Article

\title{
The Inhibitory Effect of Cordycepin on the Proliferation of MCF-7 Breast Cancer Cells, and Its Mechanism: An Investigation Using Network Pharmacology-Based Analysis
}

\author{
Dahae Lee ${ }^{1,+}$, Won-Yung Lee ${ }^{2,+}$, Kiwon Jung ${ }^{3,+} \mathbb{D}$, Yong Sam Kwon ${ }^{4}$, Daeyoung Kim ${ }^{5}$, \\ Gwi Seo Hwang ${ }^{2}$, Chang-Eop Kim ${ }^{2}$, Sullim Lee ${ }^{5, *(D)}$ and Ki Sung Kang ${ }^{2, *}$ \\ School of Pharmacy, Sungkyunkwan University, Suwon 16419, Korea \\ College of Korean Medicine, Gachon University, Seongnam 13120, Korea \\ Institute of Pharmaceutical Sciences, College of Pharmacy, CHA University, Sungnam 13844, Korea \\ Dong-A Pharmaceutical Co., LTD., Yongin 17073, Korea \\ 5 Department of Life Science, College of Bio-Nano Technology, Gachon University, Seongnam 13120, Korea \\ * $\quad$ Correspondence: sullimlee@gachon.ac.kr (S.L.); kkang@gachon.ac.kr (K.S.K.); Tel.: +82-42-750-5402 (K.S.K.) \\ + These authors contributed equally to this work.
}

Received: 28 June 2019; Accepted: 21 August 2019; Published: 26 August 2019

check for updates

\begin{abstract}
Cordyceps militaris is a well-known medicinal mushroom. It is non-toxic and has clinical health benefits including cancer inhibition. However, the anticancer effects of $C$. militaris cultured in brown rice on breast cancer have not yet been reported. In this study, we simultaneously investigated the anticancer effects of cordycepin and an extract of $C$. militaris cultured in brown rice on MCF-7 human breast cancer cells using a cell viability assay, cell staining with Hoechst 33342, and an image-based cytometric assay. The C. militaris concentrate exhibited significant MCF-7 cell inhibitory effects, and its $\mathrm{IC}_{50}$ value was $73.48 \mu \mathrm{g} / \mathrm{mL}$. Cordycepin also exhibited significant MCF-7 cell inhibitory effects, and its $\mathrm{IC}_{50}$ value was $9.58 \mu \mathrm{M}$. We applied network pharmacological analysis to predict potential targets and pathways of cordycepin. The gene set enrichment analysis showed that the targets of cordycepin are mainly associated with the hedgehog signaling, apoptosis, p53 signaling, and estrogen signaling pathways. We further verified the predicted targets related to the apoptosis pathway using western blot analysis. The C. militaris concentrate and cordycepin exhibited the ability to induce apoptotic cell death by increasing the cleavage of caspase-7 -8 , and -9 , increasing the Bcl-2-associated X protein/ B-cell lymphoma 2 (Bax/Bcl-2) protein expression ratio, and decreasing the protein expression of X-linked inhibitor of apoptosis protein (XIAP) in MCF-7 cells. Consequently, the C. militaris concentrate and cordycepin exhibited significant anticancer effects through their ability to induce apoptosis in breast cancer cells.
\end{abstract}

Keywords: Cordyceps militaris; brown rice; cordycepin; MCF-7; apoptosis

\section{Introduction}

The death rate due to breast cancer is $14 \%$, and this malignancy accounts for $23 \%$ of all cancer cases. Therefore, it remains a significant clinical and societal issue worldwide [1]. Patients diagnosed with breast cancer may receive drug therapy, surgery, hormone therapy, and/or radiotherapy. These treatments have a significant and long-term impact on patients' lives owing to the side effects of drug therapy and hormone therapy and the limitations of surgery and radiotherapy [2,3]. Among them, cisplatin is one of the most widely used chemotherapeutic drugs for clinical treatment [4]. Although the use of cisplatin causes toxic adverse effects such as nephrotoxicity, this drug is still 
widely used due to its high efficacy as an anticancer drug [5]. For this reason, interest in the development of drugs from natural products for the prevention and treatment of breast cancer has increased. Experimental studies indicate that many natural products and their bioactive compounds have anticancer effects on breast cancer [6-10]. Various mechanisms, including inhibition of cell proliferation, [6,11] apoptosis [11], cell cycle arrest, inhibition of metastasis [6], downregulation of estrogen receptor alpha (ER- $\alpha$ ) expression [7], and inhibition of angiogenesis of cells, are involved in anticancer effects on breast cancer [3]. Therefore, researchers in the past have urged continuing research on potential and effective natural products so as to identify their bioactive compounds for the treatment of breast cancer. Medicinal mushrooms such as Cordyceps species, Agaricus bisporus, Pleurotus eous, Ganoderma lucidum, and Amauroderma rude have shown anticancer effects on breast cancer both in vivo and in vitro [7,12]. Among medicinal mushrooms, Cordyceps species are considered to have various positive aspects in terms of safety (nontoxicity) [13] as well as clinical health effects such as immuno-enhancing activity [11], neuroprotective activity [14,15], anticancer effects [16], antimicrobial activity [17], and anti-inflammatory activity [18,19]. Many experimental studies have also been published on the anticancer effects of extracts of $C$. militaris in vivo and in vitro on breast cancer [20-23].

However, Cordyceps species are very rare in nature and are difficult to commercialize. Therefore, studies on Cordyceps species are not consistent because they have been studied using mycelia, wild-collected specimens, and Cordyceps cultured in insects, and other sources. Recently, the process of cultivating Cordyceps species in brown rice was successfully established, and many studies using Cordyceps species cultured in brown rice have been reported [18,24-29]. Among these, Cordyceps militaris, which is known as a representative Cordyceps species, has been cultured in brown rice, and its extract has been reported to have anticancer effects, including effects against breast cancer [25].

Many compounds such as cordycepin, mannitol, ergosterol, and polysaccharides isolated from C. militaris have been reported to have diverse pharmacological activities with anti-oxidative, anti-inflammatory, antiviral, antidiabetic, anti-platelet aggregation, and anticancer effects [30-32]. In particular, the anticancer effects of cordycepin on various cancers have been reported in many studies $[20,33,34]$. These anticancer effects of cordycepin are attributed to a structural similarity with cellular nucleosides and adenosine. Thus, cordycepin acts like a nucleoside analogue and inhibits the polyadenylation of mRNA of cancer cells $[35,36]$. While cordycepin has been examined in a number of studies related to cancer, there are no studies simultaneously examining the anticancer effects of cordycepin and C. militaris on breast cancer.

Network pharmacology has emerged as a promising approach to elucidate the systems-level mechanisms of natural products [37-39]. It understands complex diseases, including cancer, as a perturbation of interconnected complex biological networks and identifies the mechanism of drug action in terms of the network topology [40,41]. Natural products are known to exert their therapeutic effects by acting on multiple targets of multiple ingredients, and these mechanisms are well-suited to the core concept of network pharmacology. Therefore, researchers have employed network pharmacology methods to screen potent anticancer agents from natural products by predicting their potential targets and pathways [41-43].

In the present study, we investigated the anticancer effects of cordycepin and the extract of C. militaris cultured in brown rice on MCF-7 human breast cancer cells. To explore the systems-level mechanism of cordycepin, we predicted potential targets and pathways related to breast cancer using network pharmacology methods. Finally, we verified the predicted targets of cordycepin related to the apoptosis pathway in vitro.

\section{Materials and Methods}

\subsection{Cordyceps Militaris Concentrate}

Cordyceps militaris concentrate was provided by Dong-A Pharmaceutical (Yongin, Korea). Briefly, it was extracted in $50 \%$ ethanol $(v / v)$ solution in water from Cordyceps militaris cultured in brown 
rice. Then, it was concentrated under low pressure. For the in vitro and in vivo experiments, it was freeze-dried.

\subsection{Cell Culture}

The MCF-7 human breast cancer cell line, MDA-MB-231 human breast cancer cell line, LLC-PK1 pig kidney epithelial cell line and human umbilical vein endothelial (HUVEC) cell line were purchased from the American Type Culture Collection (ATCC, Manassas, VA, USA). MCF-7 and MDA-MB-231 cells were grown in Roswell Park Memorial Institute 1640 medium (RPMI 1640) (Corning, Manassas, VA, USA). LLC-PK1 cells were grown in Dulbecco's modified Eagle's medium (Corning, Manassas, VA, USA). Both media types contained 10\% fetal bovine serum (Gibco BRL, Carlsbad, MD, USA), 100 units/mL penicillin, and $100 \mathrm{mg} / \mathrm{mL}$ streptomycin. HUVECs were grown in Clonetics EGM-2 MV Microvascular Endothelial BulletKit (Lonza Inc., Walkersville, MD, USA). Cultures were maintained at $37^{\circ} \mathrm{C}$ in a humidified atmosphere with $5 \% \mathrm{CO}_{2}$.

\subsection{Determination of Cell Viability}

The cell viability of MCF-7 cells in C. militaris concentrate and cordycepin was evaluated using an Ez-Cytox Cell Viability Assay Kit (Dail Lab Service Co., Seoul, Korea). Briefly, cells with a density of $1 \times 10^{4}$ cells/100 $\mu \mathrm{L}$ were seeded onto 96-well plates. After incubation for $24 \mathrm{~h}, \mathrm{C}$. militaris concentrate and cordycepin at various concentrations were added. After treatment for $24 \mathrm{~h}, 10 \mu \mathrm{L}$ of Ez-Cytox solution was added and incubated for $30 \mathrm{~min}$. The absorbance was measured at $450 \mathrm{~nm}$ (absorbance for live cells) in a microplate reader (PowerWave XS; Bio-Tek Instruments, Winooski, VT, USA). Cisplatin, which is one of the most widely used chemotherapeutic drug for breast cancer, was used as a positive control.

\subsection{Cell Staining with Hoechst 33342}

The nuclear condensation of MCF-7 cells induced by C. militaris concentrate and cordycepin was evaluated using Hoechst 33342 staining (Sigma Aldrich, St. Louis, MO, USA). Briefly, cells at a density of $4 \times 10^{5}$ cells $/ 3 \mathrm{~mL}$ were seeded onto 6-well plates. After incubation for $24 \mathrm{~h}, \mathrm{C}$. militaris concentrate and cordycepin at various concentrations were added. After treatment for $24 \mathrm{~h}, 2 \mu \mathrm{L}$ of Hoechst 33342 solution was added, and the plates were then incubated for $10 \mathrm{~min}$. Subsequently, the stained cells were analyzed using an IX50 fluorescent microscope equipped with a charge-coupled device (CCD) camera (Olympus, Tokyo, Japan).

\subsection{Image-Based Cytometric Assay}

The apoptotic death of MCF-7 cells induced by C. militaris concentrate and cordycepin was evaluated using an image-based cytometric assay kit (Invitrogen, Temecula, CA, USA). Briefly, cells at a density of $4 \times 10^{5}$ cells $/ 3 \mathrm{~mL}$ were seeded onto 6-well plates. After incubation for $24 \mathrm{~h}, \mathrm{C}$. militaris concentrate and cordycepin at various concentrations were added. After treatment for $24 \mathrm{~h}$, cells were collected and suspended in Annexin V Binding Buffer (Life Technologies, Carlsbad, CA, USA). Then, Annexin V Alexa Fluor 488 (Invitrogen) was added and the cells were incubated in the dark for $30 \mathrm{~min}$ at $20 \pm 5{ }^{\circ} \mathrm{C}$. Next, apoptotic cells stained with green Annexin V Alexa Fluor 488 were counted and analyzed with a Tali image-based cytometer (Invitrogen).

\subsection{Western Blot Analysis}

The signaling pathways of MCF-7 cell apoptosis induced by C. militaris concentrate and cordycepin were evaluated using western blot analysis. Briefly, cells at a density of $4 \times 10^{5}$ cells $/ 3 \mathrm{~mL}$ were seeded onto 6-well plates. After incubation for 24 h, C. militaris concentrate and cordycepin at various concentrations were added. After treatment for $24 \mathrm{~h}$, cells were collected using a cell scraper and lysed using radio immunoprecipitation assay (RIPA) buffer (Cell Signaling Technology, Inc., MA, 
USA) containing $1 \times$ ethylenediaminetetraacetic acid (EDTA)-free protease inhibitor cocktail and $1 \mathrm{mM}$ phenylmethylsulfonyl fluoride (PMSF). The protein concentrations of samples were determined using a Pierce BCA Protein Assay Kit (Thermo Scientific, Carlsbad, CA, USA). Protein (20 $\mu$ P) was mixed with $4 \times$ NuPAGE LDS Sample Buffer (Thermo Scientific, Carlsbad, CA, USA). After boiling for 10 min, the proteins were separated on precast $4-15 \%$ Mini-PROTEAN TGX (Tris-Glycine. eXtended) gel (Bio-Rad, Hercules, CA, USA) and then electrotransferred onto polyvinylidene fluoride (PVDF) transfer membranes. To enhance the detection of proteins, membranes were incubated with specific primary antibodies to Bcl-2-associated X protein (Bax), B-cell lymphoma 2 (Bcl-2), cleaved caspase-7, cleaved caspase-8, X-linked inhibitor of apoptosis protein (XIAP), and glyceraldehyde 3-phosphate dehydrogenase (GAPDH) (Cell Signaling Technology, Inc.), followed by incubation with horseradish peroxidase-conjugated secondary goat anti-rabbit antibody (Cell Signaling Technology, Inc.). The bound antibodies were visualized using Pierce ECL (enhanced chemiluminescence) Western Blotting Substrate (Rockford, IL, USA) and a FUSION Solo Chemiluminescence System (PEQLAB Biotechnologie $\mathrm{GmbH}$, Erlangen, Germany).

\subsection{Network Pharmacological Analysis}

Network pharmacological analysis was performed by predicting targets of ingredients in traditional Chinese medicine (TCM) and constructing a compound-target network. The potential targets of cordycepin were obtained from the TCM-mesh (http://mesh.tcm.microbioinformatics.org) based on the combined score [44]. The combined score is calculated by a model that predicts drug-target interaction using random forests [45]. To get as many potential targets of cordycepin as possible, we set the threshold of the combined score to 150 (minimal threshold suggested by TCM-mesh). Then, a compound-target network was constructed by linking herbal ingredients and their predicted targets using the drug-target interaction information.

Gene set enrichment analysis (GSEA) based on the Kyoto Encyclopedia of Genes and Genomes (KEGG) database was performed to identify potential pathways related to breast cancer using Enrichr [46]. Enrichr computes enrichment by assessing multiple gene-set libraries (e.g., gene ontology, KEGG, and Online Mendelian Inheritance in Man (OMIM)) and calculates adjusted $p$-values, Z-scores and combined scores for the gene lists of interest (target genes). The combined score is calculated by the logarithm of multiplication of the $p$-value and z-score (note that this combined score is different from the combined score in the TCM-mesh).

\subsection{Statistical Analysis}

The data are presented as the mean \pm standard deviation (SD). Statistical significance was determined using the Student's $t$-test and the hypergeometric test. $p$-Values less than 0.05 were considered statistically significant.

\section{Results}

\subsection{Effects of Cordyceps Militaris Concentrate and Cordycepin on MCF-7 Breast Cancer Cell Viability}

Cell viability assays were performed to evaluate the cytotoxic effects of the $C$. militaris concentrate and cordycepin on MCF-7 cells. Cisplatin was used as the positive control. C. militaris concentrate suppressed cell proliferation in a concentration-dependent manner (Figure 1A, $\mathrm{IC}_{50}: 73.48 \pm 2.76 \mu \mathrm{g} / \mathrm{mL}$ ). Cordycepin also significantly suppressed cell proliferation in a concentration-dependent manner (Figure 1B, $\mathrm{IC}_{50}: 9.58 \pm 0.99 \mu \mathrm{M}$ ). Cisplatin suppressed cell proliferation in a concentration-dependent manner, but its $\mathrm{IC}_{50}$ value was higher than that of cordycepin (Figure $1 \mathrm{C} \mathrm{IC}_{50}: 48.50 \pm 1.84 \mu \mathrm{M}$ ). 
(A)

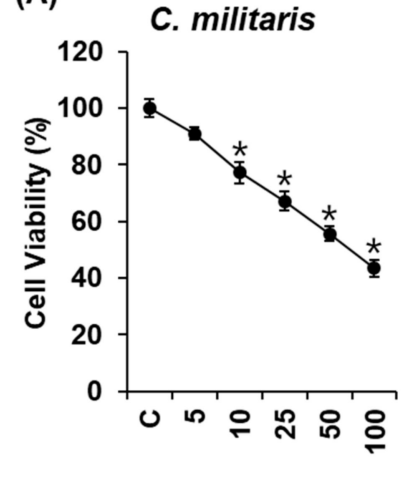

Concentration $(\mu \mathrm{g} / \mathrm{mL})$
(B)

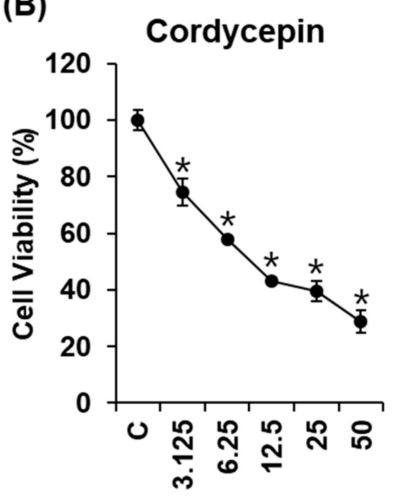

Concentration $(\mu \mathrm{M})$
(C)

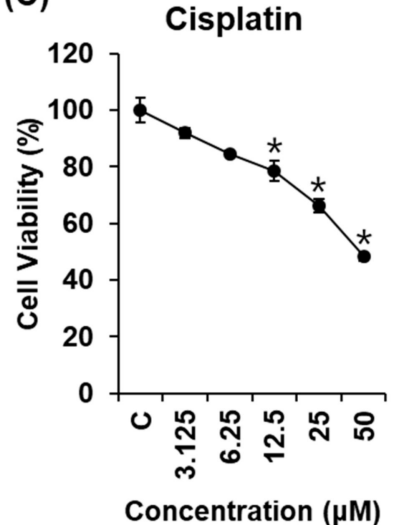

(D)

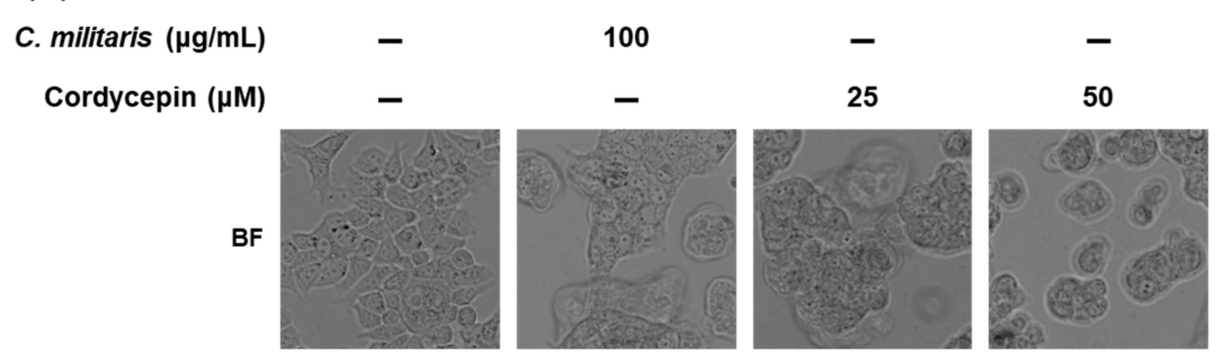

Figure 1. Effects of Cordyceps militaris concentrate and cordycepin on MCF-7 breast cancer cell viability. Cytotoxic effects of (A) C. militaris concentrate, (B) cordycepin, and (C) cisplatin on MCF-7 cells. (D) Effects of $C$. militaris concentrate and cordycepin on morphological changes in MCF-7 cells. Data are the means of experiments performed in triplicate. Data are presented as the mean \pm standard deviation (SD). and were analyzed using the Student's $t$-test. ${ }^{*} p<0.05$ versus non-treated cells.

Based on these results, we investigated the effects of $C$. militaris concentrate and cordycepin on morphological changes, which contributed to the inhibition of MCF-7 cell viability, using a phase contrast inverted microscope and phase contrast fluorescence microscope (Figure 1D). After treatment with C. militaris $(100 \mu \mathrm{g} / \mathrm{mL})$ and cordycepin $(25,50 \mu \mathrm{M})$, most cells detached from the cell culture plate, and apoptotic cell morphology such as membrane blebbing, cell shrinkage, and cell condensation [47] increased in comparison to the untreated normal cells.

\subsection{Network Pharmacological Analysis of Cordycepin}

Network pharmacological analysis was conducted to elucidate the systems-level mechanism of cordycepin. We constructed and visualized the compound-target network of cordycepin using Cytoscape [48]. In the compound-target network, nodes represented cordycepin and its targets, whereas edges represented the predicted interactions between cordycepin and targets. The targets of cordycepin were colored to indicate the pathways related to breast cancer. The pathways related to breast cancer obtained from literature were as follows: apoptosis pathway, breast tumor kinase pathway, cyclin-dependent kinases pathway, estrogen signaling pathway, hedgehog signaling pathway, human epidermal growth factor receptor 2 (HER2) signaling pathway, mammalian target of rapamycin signaling pathway, notch signaling pathway, phosphoinositide 3-kinases (PI3K)- protein kinase B (Akt) signaling pathway, and Wingless and INT- 1 (Wnt)/ $\beta$-catenin signaling pathway $[49,50]$. The numbers of related targets for the apoptosis, estrogen signaling, notch signaling, and hedgehog signaling pathways were 5, 2, 2, and 2, respectively (Figure 2). B-cell lymphoma 2 (BCL2) was simultaneously related to the apoptosis, estrogen signaling, and hedgehog signaling pathways. 


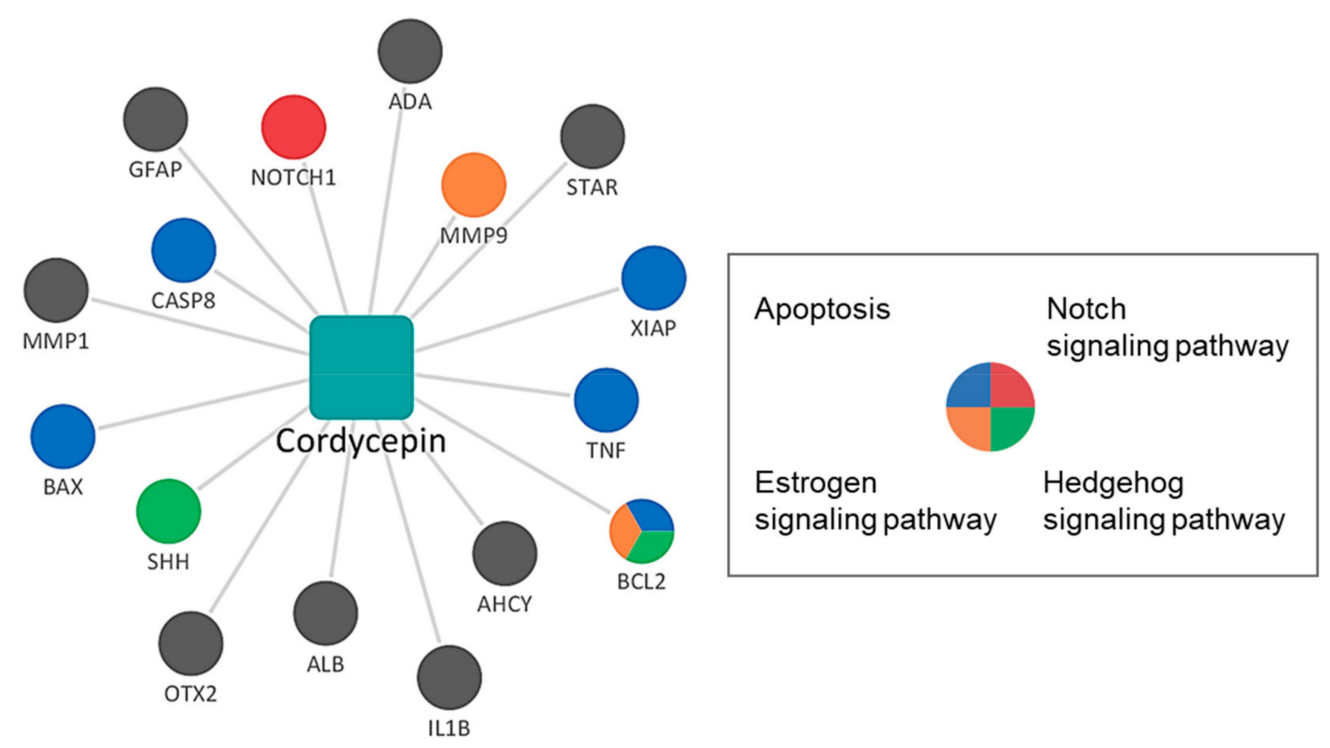

Figure 2. Compound-target network of Cordyceps militaris. Rectangles represent the compounds, and circles represent the targets. Nodes related to apoptosis, estrogen, hedgehog, and notch signaling pathway in KEGG and validated targets by western blotting analysis are colored. ADA, adenosine deaminase; AHCY, adenosylhomocysteinase; ALB, albumin; BAX, BCL2 associated X, apoptosis regulator; BCL2, BCL2 apoptosis regulator; CASP8, caspase 8; GFAP, glial fibrillary acidic protein; IL1B, interleukin 1 beta; MMP1, matrix metallopeptidase 1; MMP9, matrix metalloproteinase-9; NOTCH1, notch receptor 1; OTX2, orthodenticle homeobox 2; $\mathrm{SHH}$, sonic hedgehog signaling molecule; STAR, steroidogenic acute regulatory protein; TNF, tumor necrosis factor; XIAP, X-linked inhibitor of apoptosis.

Next, we conducted GSEA to identify potential pathways that are significantly associated with the targets of cordycepin among the pathways related to breast cancer. The results showed that the apoptosis pathway had the highest overlap and lowest $p$-value for the targets of cordycepin. In addition, the hedgehog signaling, p53 signaling, and estrogen signaling pathways were significantly associated with the targets of cordycepin (Table 1).

Table 1. Significant enrichment pathway related to breast cancer by targets of cordycepin (adjusted $p$-value of $\leq 0.05)$.

\begin{tabular}{cccccc}
\hline Term & Overlap & Adjusted $p$-Value & Z-Score & Combined Score & Genes \\
\hline $\begin{array}{c}\text { Hedgehog signaling } \\
\text { pathway }\end{array}$ & $2 / 47$ & 0.002 & -55.763 & 410.509 & SHH, BCL2 \\
Apoptosis & $5 / 143$ & $>0.001$ & -4.979 & 81.932 & CASP8, BCL2, BAX, \\
P53 signaling pathway & $3 / 72$ & $>0.001$ & -6.464 & 68.700 & CASP8, BCL2, BAX \\
$\begin{array}{c}\text { Estrogen signaling } \\
\text { pathway }\end{array}$ & $2 / 137$ & 0.011 & -0.946 & 4.964 & BCL2, MMP9 \\
\hline
\end{tabular}

\subsection{Effects of the Cordyceps Militaris Concentrate and Cordycepin on Apoptosis}

To verify the anticancer mechanisms identified through network pharmacological analysis, image-based cytometric assay and western blot analysis were performed for the $C$. militaris concentrate and cordycepin to evaluate their apoptotic effect on MCF-7 cells. To explore the effects of $C$. militaris and cordycepin on apoptotic cell death, MCF-7 cells were stained with Annexin V. After treatment with $C$. militaris concentrate $(100 \mu \mathrm{g} / \mathrm{mL})$ and cordycepin $(25,50 \mu \mathrm{M})$, apoptotic cells stained with enhanced Annexin V (green fluorescence) increased as compared to untreated normal cells (Figure 3A). The percentage of apoptotic cells was significantly increased to $22.50 \pm 2.17 \%, 40.06 \pm 3.49 \%$, and $52.63 \pm 2.56 \%$ after treatment with C. militaris concentrate $(100 \mu \mathrm{g} / \mathrm{mL})$ and cordycepin $(25 \mu \mathrm{M}, 50 \mu \mathrm{M})$, respectively (Figure 3B). 
(A)

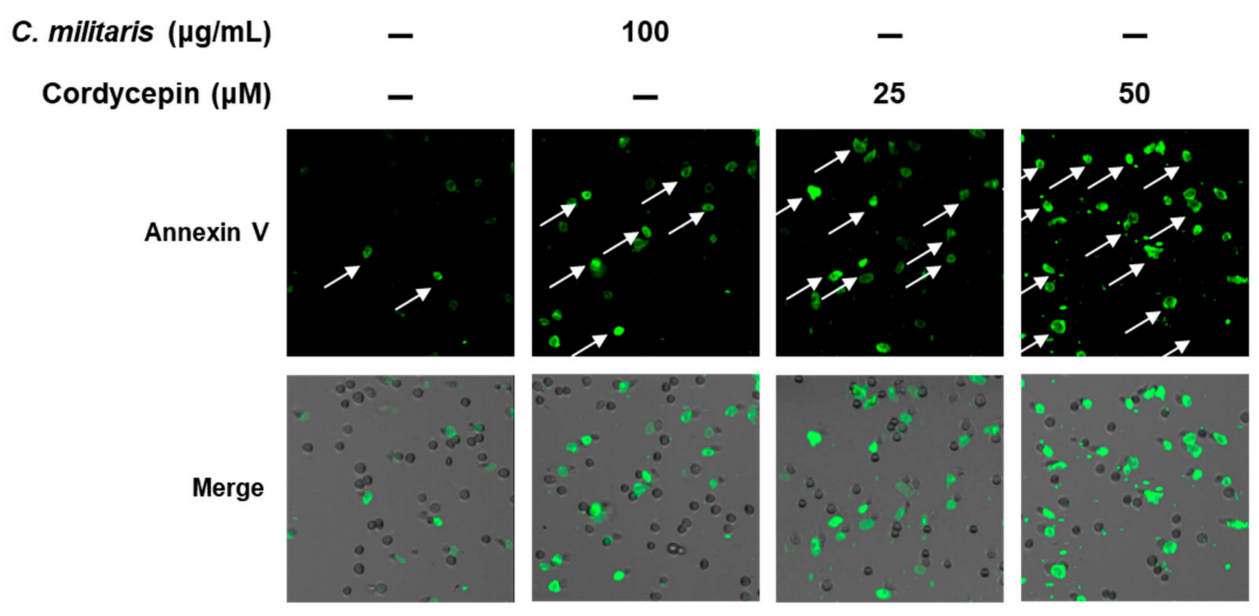

(B)

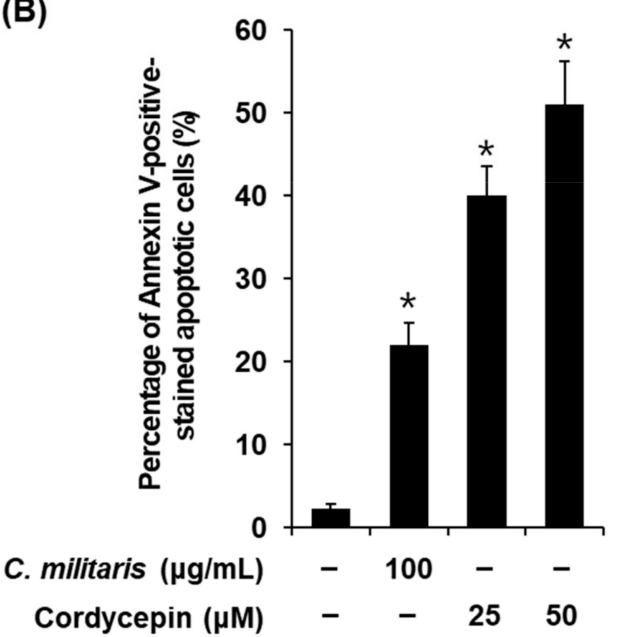

Figure 3. Effects of Cordyceps militaris concentrate $(100 \mu \mathrm{g} / \mathrm{mL})$ and cordycepin $(25$ and $50 \mu \mathrm{M})$ on apoptosis in MCF-7 breast cancer cells exposed for $24 \mathrm{~h}$ by image-based cytometric assay. (A) Representative images for apoptosis detection; (B) Percentage of Annexin V-positive-stained apoptotic cells. Data are the means of experiments performed in triplicate. Data are presented as the mean \pm SD. and were analyzed using the Student's $t$-test. ${ }^{*} p<0.05$ versus non treated cells.

In keeping with these results, DNA fragmentation identifying apoptotic cells was increased after treatment with $C$. militaris concentrate $(100 \mu \mathrm{g} / \mathrm{mL})$ and cordycepin $(25,50 \mu \mathrm{M})$. Blue fluorescence of Hoechst 33342 on these cells was brighter than that of untreated normal cells (Figure 4A). To identify the apoptotic mechanism of $C$. militaris concentrate and cordycepin, western blot analysis was performed. After treatment with $C$. militaris concentrate $(100 \mu \mathrm{g} / \mathrm{mL})$ and cordycepin $(25,50 \mu \mathrm{M})$, the Bax (pro-apoptotic Bcl-2 family)/Bcl-2 (anti-apoptotic Bcl-2 family) ratio was increased by more than two times. And cleavage of caspase- 8 , which is an apoptotic initiator, was significantly increased by more than five times. Furthermore, cleavage of caspase-7, an apoptotic effector caspase, was significantly increased by more than three times. Meanwhile, treatment of cordycepin inhibited XIAP, which is the X-linked inhibitor of apoptosis protein (Figure 4B). 
(A)

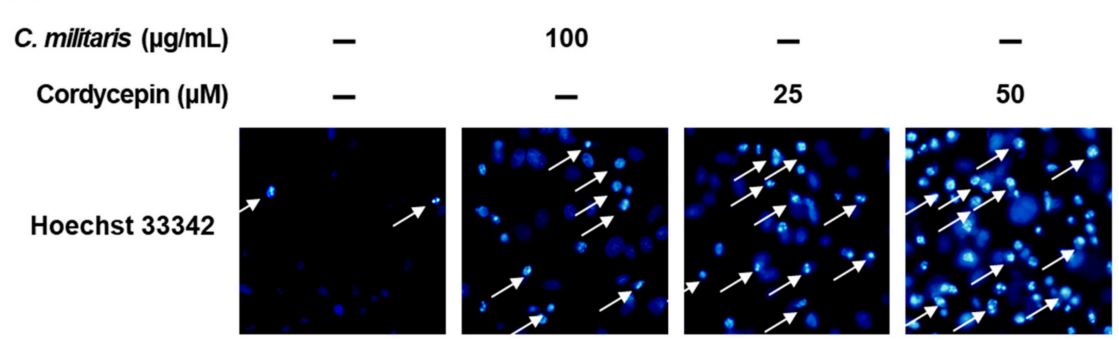

(B)
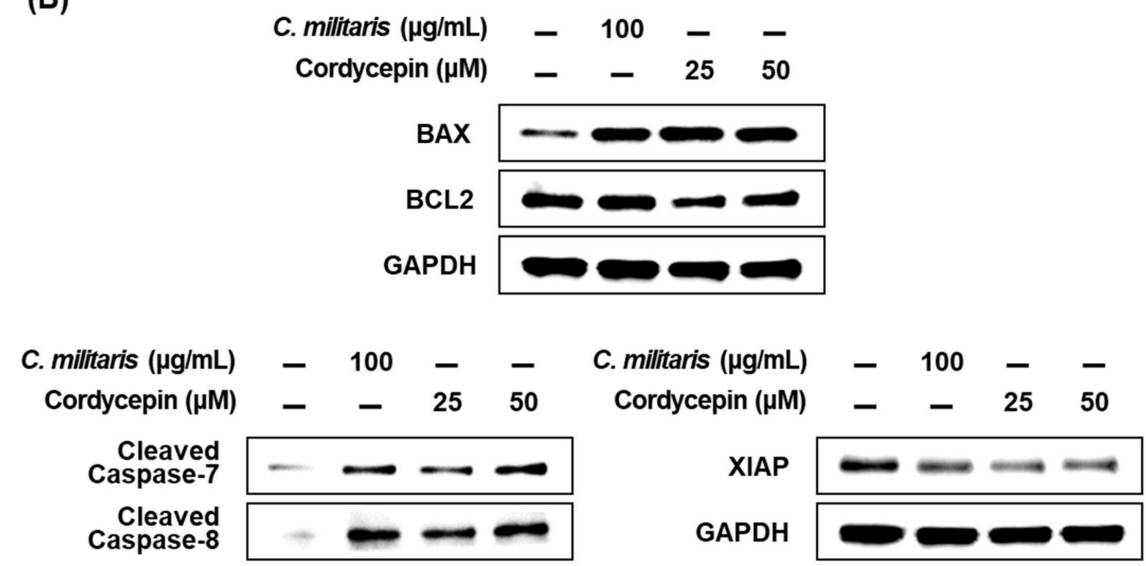

GAPDH

(C)

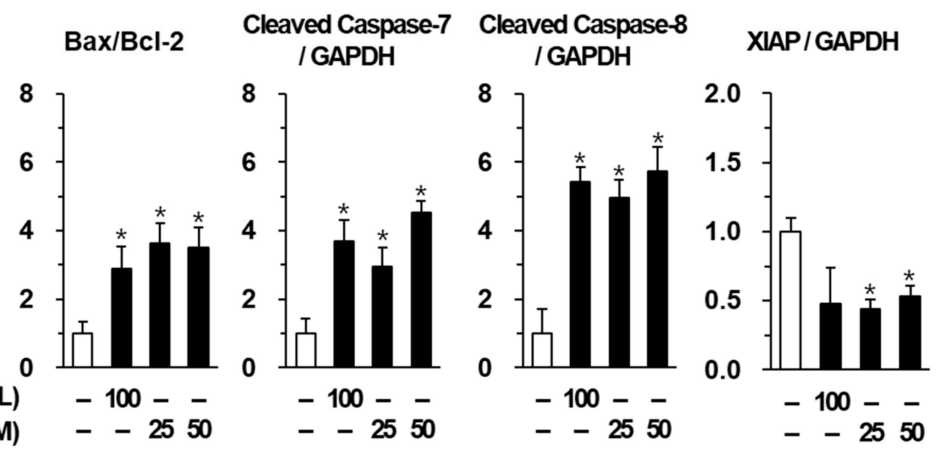

Figure 4. Effects of Cordyceps militaris concentrate $(100 \mu \mathrm{g} / \mathrm{mL})$ and cordycepin $(25$ and $50 \mu \mathrm{M})$ on apoptosis in MCF-7 breast cancer cells exposed for $24 \mathrm{~h}$. (A) Results of Hoechst 33342 fluorescent staining of Cordyceps militaris and cordycepin to detect nuclear condensation of MCF-7 cells. (B) Protein expression of Bax, Bcl-2, cleaved caspase-7, cleaved caspase-8, XIAP and GAPDH. (C) Graph of relative protein expression. Data are the means of experiments performed in triplicate. Data are presented as the mean $\pm \mathrm{SD}$. and were analyzed using the Student's $t$-test. ${ }^{*} p<0.05$ versus non-treated cells.

\section{Discussions}

In our investigations on the Cordyceps militaris concentrate and cordycepin to examine in vitro anticancer activity on MCF-7 human breast cancer cells, we observed that both the C. militaris concentrate and cordycepin exhibited a significant cytotoxic effect on MCF-7 cells. The $\mathrm{IC}_{50}$ value of the latter was about five times lower than that of cisplatin, which is currently the most effective platinum-based drug for breast cancer [51]. The extract of $C$. militaris exhibited cytotoxic effects on the $4 \mathrm{~T} 1$ murine breast cancer cell line in a recent study, and the extract $(100,200$, and $400 \mu \mathrm{g} / \mathrm{mL})$ inhibited cell viability in a concentration-dependent manner [52]. In addition, cordycepin $(100 \mu \mathrm{M})$ has been reported to inhibit cell viability below 50\% in MCF-7, MDA-MB 231, and MDA-MB-435 human breast 
cancer cells [53]. These results indicate that cordycepin exhibits cytotoxic effects on various types of breast cancer cell lines.

In the present study, the C. militaris concentrate and breast cancer cell line also suppressed cell proliferation of the MDA-MB-231 breast cancer cell line in a concentration-dependent manner (Figure S1). Although active concentrations on MDA-MB-231 were higher than on MCF-7, C. militaris concentrate and cordycepin also inhibited cell proliferation of the MDA-MB-231 breast cancer cell line. Meanwhile, $100 \mu \mathrm{g} / \mathrm{mL}$ of $C$. militaris concentrate did not affect the viabilities of LLC-PK1 pig kidney epithelial cells and human umbilical vein endothelial (HUVEC) cells, which are normal cell lines (Figure S2). Cordycepin also did not affect the viabilities of LLC-PK1 and HUVEC cells until $50 \mu \mathrm{M}$. We suggest that they are good candidates for targeting breast cancer.

To investigate the detailed cytotoxic effects, a phase contrast inverted microscope was used to evaluate the pattern of cell death in MCF-7 cells. Results of this observation indicated that apoptotic cell morphologies including membrane blebbing, cell shrinkage, and cell condensation were increased after treatment with $C$. militaris concentrate and cordycepin. These findings were consistent with the results of a previous study, which reported that cell debris and irregularly shaped cells were increased in MCF-7 cells after treatment with cordycepin $(100 \mu \mathrm{M})$ [54]. However, these are hallmarks of apoptosis but not exact evidence [47]. Therefore, to evaluate apoptotic cell death under treatment with $C$. militaris concentrate and cordycepin in MCF-7 cells, Hoechst 33342 staining and image-based cytometric assays were used to distinguish apoptotic cells from non-apoptotic cells. Observation with a phase-contrast fluorescence microscope indicated that blue fluorescence from Hoechst 33342-staining of DNA as well as DNA fragmentation, which is a key characteristic of apoptosis [55], were also increased. In keeping with this result, apoptotic cells stained with enhanced Annexin V (green fluorescence) were increased after treatment with C. militaris concentrate and cordycepin.

To elucidate the systems-level mechanism, a compound-target network was constructed based on the predicted targets of cordycepin. The predicted targets were associated with the apoptosis, estrogen signaling, hedgehog signaling, and notch signaling pathways. In addition, GSEA was used to identify pathways that were significantly enriched in the target list of cordycepin. Among the pathways associated with breast cancer, the apoptosis pathway showed the greatest overlap, lowest $p$-value, and highest combined score.

To further investigate the changes in protein expression involving the apoptotic pathways, western blot analysis was used. The apoptotic pathways involved in the anticancer effects of cordycepin have been extensively studied in various cancers over a long period of time (about 60 years) [36]. Collectively, treatment with cordycepin increases the phosphorylation of terminal deoxynucleotidyl transferase (TdT) by protein kinase A (PKA) [56]. Treatment with cordycepin also induces apoptosis through the mitochondria-mediated intrinsic apoptotic pathways indicated by translocation of Bax from the cytosol to the mitochondria, release of cytochrome $c$ from the mitochondria to the cytosol, and activation of caspase-9. Caspase- 9 is activated by apaf- 1 (apoptosome) and activates caspase- 3 and $-7[57,58]$. In addition, treatment with cordycepin induces death receptor-mediated extrinsic apoptotic pathways, as indicated by the activation of caspase- 8 , which stimulates two parallel cascades. One is the activation of caspase- 3 and -7 and the other is the release of cytochrome $c$ induced by the truncated Bid (pro-apoptotic Bcl-2 family), which translocates to the mitochondria and results in an increase in the Bax/Bcl-2 ratio [59,60]. Furthermore, treatment of cordycepin inhibited XIAP. XIAP is bound to caspase- $3,-7$, and -9 and suppresses cell death by caspase overproduction [61].

However, the MCF-7 cells used in our study were caspase-3 deficient [62,63]. Therefore, caspase-7 was confirmed as the apoptotic effector caspase. In the MCF-7 cells, C. militaris concentrate and cordycepin induced the cleavage of caspase-8, which are apoptotic initiator caspases. This resulted in the cleavage of caspase-7, an apoptotic effector caspase, and an increase in the Bax/Bcl-2 ratio. These results suggest that the $C$. militaris concentrate and cordycepin induced apoptosis via both mitochondrial-mediated intrinsic, and death receptor-mediated extrinsic apoptotic pathways. The targets of cordycepin related to the apoptosis pathway were summarized using KEGG Mapper (Figure 5). 


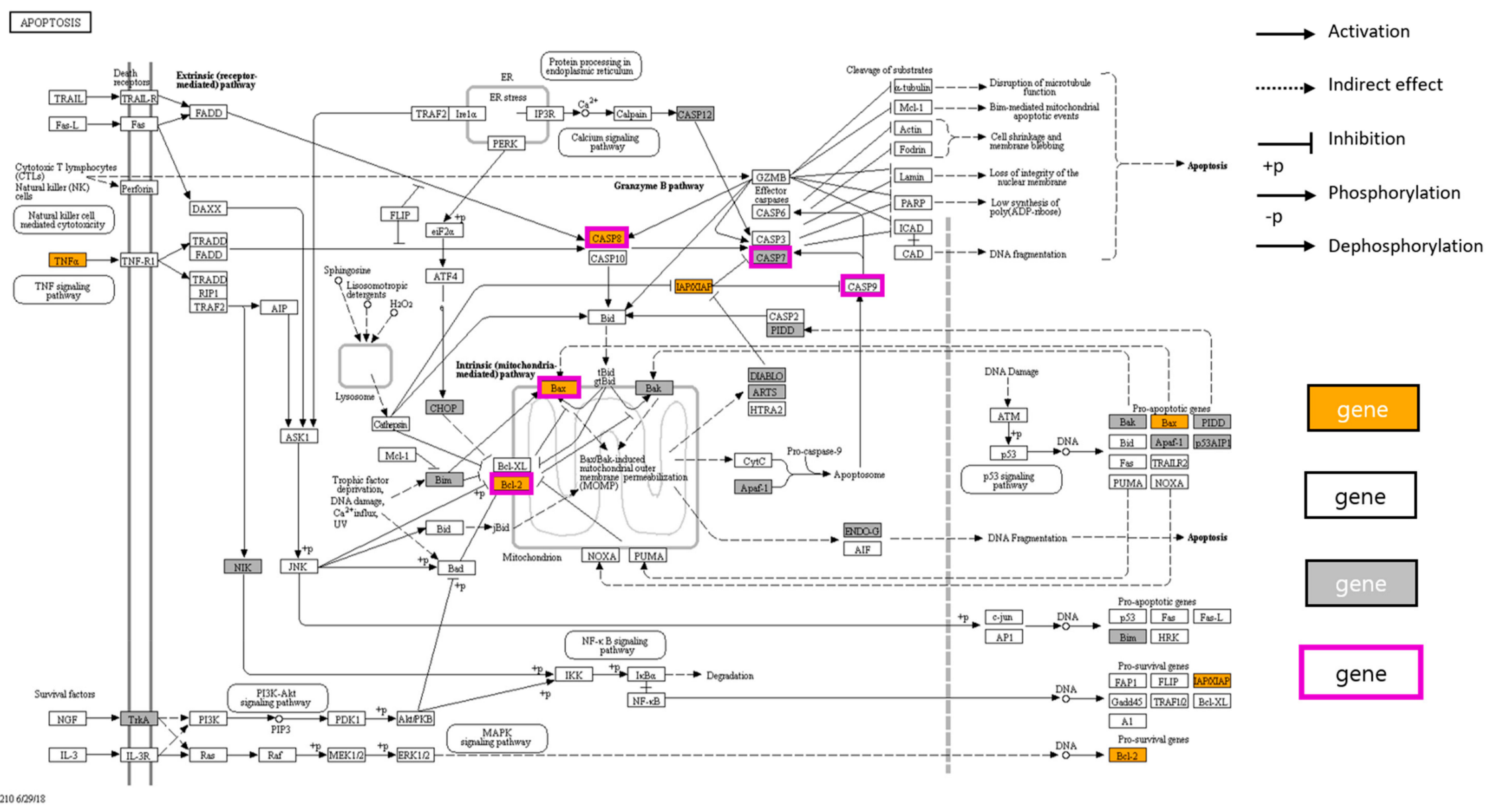

Figure 5. Apoptosis pathways (hsa04210) and targets of cordycepin. The pathway maps were constructed using KEGG mapper. The orange colored box represents a predicted target to interact cordycepin, the uncolored box represents a target that is expected to not interact, the gray colored box represents a target which is outside the predictable target list in TCM-mesh, and the purple-bordered box represents a validated target by western blot. 
Among the targets of cordycepin investigated by western blot analysis, three target genes were also found in the predicted target lists (CASP8, Bax, Bcl-2 and XIAP). The accordance rate was significant ( $p$-value $<0.001$, hypergeometric test), which supports the reliability of the predicted results. Of the two mismatched targets, CASP9 was predicted not to interact with cordycepin (incorrect prediction), and CASP7 was not found in the predicted target list of TCM-mesh (unpredictable target).

In summary, we performed several in vitro experimental methods including cell viability assays, cell staining with Hoechst 33342, image-based cytometric assays, and western blot analysis to evaluate the apoptotic effects of the $C$. militaris concentrate and cordycepin on MCF-7 human breast cancer cells. The $C$. militaris concentrate and cordycepin exhibited potent cytotoxic effects on MCF-7 cells and could increase the cleavage of caspase-7 -8, and -9 , and increase the Bax/Bcl-2 protein expression ratio in MCF-7 cells. Additionally, we applied network pharmacological analysis to predict potential targets and pathways of cordycepin and the targets related to the apoptosis pathway were validated in vitro.

\section{Conclusions}

In this study, the C. militaris concentrate cultured in brown rice and cordycepin isolated from C. militaris induced the cell death of MCF-7 human breast cancer cells. Network pharmacological analysis revealed a systems-level mechanism of cordycepin that could explain its suppressive effect on the proliferation of breast cancer cells, and the targets associated with the apoptosis pathway were verified by further experiments. This study provides a promising potential application not only for C. militaris cultured in brown rice itself but also for cordycepin isolated from C. militaris as a traditional medicine and alternative to chemotherapy in breast cancer. It also suggests that the strategy of integration of network pharmacology and experimental validation is a powerful tool to obtain a deep understanding of the mechanisms of natural products that act on multiple targets.

Supplementary Materials: The following are available online at http://www.mdpi.com/2218-273X/9/9/414/s1, Figure S1: Effects of Cordyceps militaris concentrate and cordycepin on MDA MB-231 breast cancer cell viability, Figure S2: Effects of Cordyceps militaris concentrate and cordycepin on viability of (A) LLC-PK1 pig kidney epithelial cells and (B) Human umbilical vein endothelial (HUVEC) cells.

Author Contributions: K.S.K., S.L. and K.J. designed the research. D.L., W.-Y.L., Y.S.K. and S.L. performed the experiments. D.L., W.-Y.L. and S.L. analyzed the data. K.J., D.K., G.S.H., C.-E.K. and K.S.K. interpreted the results of the experiments. W.-Y.L., S.L. and K.S.K drafted the manuscript.

Funding: This work was supported by the Korea Institute of Planning and Evaluation for Technology in Food, Agriculture, Forestry (IPET) through Agri-Bio industry Technology Development Program, funded by Ministry of Agriculture, Food and Rural Affairs (MAFRA) (316025-05-4-SB010). This work was also supported by the Basic Science Research Program of the National Research Foundation of Korea (NRF), funded by the Ministry of Science, ICT \& Future Planning (2019R1F1A1059173).

Conflicts of Interest: The authors declare no conflict of interest.

\section{References}

1. Siegel, R.L.; Miller, K.D.; Jemal, A. Cancer statistics, 2019. CA Cancer J. Clin. 2019, 69, 7-34. [CrossRef] [PubMed]

2. Waks, A.G.; Winer, E.P. Breast cancer treatment: A review. Jama 2019, 321, 288-300. [CrossRef] [PubMed]

3. Li, Y.; Li, S.; Meng, X.; Gan, R.Y.; Zhang, J.J.; Li, H.B. Dietary Natural Products for Prevention and Treatment of Breast Cancer. Nutrients 2017, 9, 728. [CrossRef] [PubMed]

4. Zhao, Y.; Jing, Z.; Li, Y.; Mao, W. Berberine in combination with cisplatin suppresses breast cancer cell growth through induction of DNA breaks and caspase-3-dependent apoptosis. Oncol. Rep. 2016, 36, 567-572. [CrossRef] [PubMed]

5. Qi, L.; Luo, Q.; Zhang, Y.; Jia, F.; Zhao, Y.; Wang, F. Advances in Toxicological Research of the Anticancer Drug Cisplatin. Chem. Res. Toxicol. 2019, 32, 469-1486. [CrossRef] [PubMed]

6. Ham, S.L.; Nasrollahi, S.; Shah, K.N.; Soltisz, A.; Paruchuri, S.; Yun, Y.H.; Luker, G.D.; Bishayee, A.; Tavana, H. Phytochemicals potently inhibit migration of metastatic breast cancer cells. Integr. Biol. (Camb) 2015, 7, 792-800. [CrossRef] [PubMed] 
7. Bak, M.J.; Das Gupta, S.; Wahler, J.; Suh, N. Role of dietary bioactive natural products in estrogen receptor-positive breast cancer. Semin. Cancer Biol. 2016, 40-41, 170-191. [CrossRef]

8. Goodarzi, S.; Tabatabaei, M.J.; Mohammad Jafari, R.; Shemirani, F.; Tavakoli, S.; Mofasseri, M.; Tofighi, Z. Cuminum cyminum fruits as source of luteolin-7-O-glucoside, potent cytotoxic flavonoid against breast cancer cell lines. Nat. Prod. Res. 2018, 22, 1-5. [CrossRef]

9. Le, T.V.T.; Nguyen, P.H.; Choi, H.S.; Yang, J.L.; Kang, K.W.; Ahn, S.G.; Oh, W.K. Diarylbutane-type Lignans from Myristica fragrans (Nutmeg) show the Cytotoxicity against Breast Cancer Cells through Activation of AMP-activated Protein Kinase. Nat. Prod. Sci. 2017, 23, 21-28. [CrossRef]

10. Yang, E.J.; An, J.H.; Son, Y.K.; Yeo, J.H.; Song, K.S. The Cytotoxic Constituents of Betula platyphylla and their Effects on Human Lung A549 Cancer Cells. Nat. Prod. Sci. 2018, 24, 219-224. [CrossRef]

11. Yang, B.; Zhu, R.; Tian, S.; Wang, Y.; Lou, S.; Zhao, H. Jatamanvaltrate P induces cell cycle arrest, apoptosis and autophagy in human breast cancer cells in vitro and in vivo. Biomed. Pharmacother. 2017, 89, 1027-1036. [CrossRef]

12. Li, J.Y.; Zou, L.; Chen, W.; Zhu, B.B.; Shen, N.; Ke, J.T.; Lou, J.; Song, R.R.; Zhong, R.; Miao, X.P. Dietary Mushroom Intake May Reduce the Risk of Breast Cancer: Evidence from a Meta-Analysis of Observational Studies. PLoS ONE 2014, 9, e93437. [CrossRef]

13. Tuli, H.S.; Sandhu, S.S.; Sharma, A.K. Pharmacological and therapeutic potential of Cordyceps with special reference to Cordycepin. 3 Biotech 2014, 4,1-12. [CrossRef]

14. Olatunji, O.J.; Feng, Y.; Olatunji, O.O.; Tang, J.; Ouyang, Z.; Su, Z.; Wang, D.; Yu, X. Neuroprotective effects of adenosine isolated from Cordyceps cicadae against oxidative and ER stress damages induced by glutamate in PC12 cells. Environ. Toxicol. Pharmacol. 2016, 44, 53-61. [CrossRef]

15. Olatunji, O.J.; Feng, Y.; Olatunji, O.O.; Tang, J.; Wei, Y.; Ouyang, Z.; Su, Z. Polysaccharides purified from Cordyceps cicadae protects PC12 cells against glutamate-induced oxidative damage. Carbohydr. Polym. 2016, 153, 187-195. [CrossRef]

16. Joshi, M.; Sagar, A.; Kanwar, S.S.; Singh, S. Anticancer, antibacterial and antioxidant activities of Cordyceps militaris. Indian J. Exp. Biol. 2019, 57, 15-20.

17. Mehrotra, S.; Kirar, V.; Vats, P.; Nandi, S.P.; Negi, P.; Misra, K. Phytochemical and antimicrobial activities of Himalayan Cordyceps sinensis (Berk.) Sacc. Indian J. Exp. Biol. 2015, 53, 36-43.

18. Chiu, C.P.; Liu, S.C.; Tang, C.H.; Chan, Y.; El-Shazly, M.; Lee, C.L.; Du, Y.C.; Wu, T.Y.; Chang, F.R.; Wu, Y.C. Anti-inflammatory cerebrosides from cultivated Cordyceps militaris. J. Agric. Food Chem. 2016, 64, 1540-1548. [CrossRef]

19. Kim, Y.S.; Shin, W.B.; Dong, X.; Kim, E.K.; Nawarathna, W.P.A.S.; Kim, H.; Park, P.J. Anti-inflammatory effect of the extract from fermented Asterina pectinifera with Cordyceps militaris mycelia in LPS-induced RAW264. 7 macrophages. Food Sci. Biotechnol. 2017, 26, 1633-1640. [CrossRef]

20. Jin, Y.; Meng, X.; Qiu, Z.; Su, Y.; Yu, P.; Qu, P. Anti-tumor and anti-metastatic roles of cordycepin, one bioactive compound of Cordyceps militaris. Saudi J. Biol. Sci. 2018, 25, 991-995. [CrossRef]

21. Kim, Y.S.; Kim, E.K.; Tang, Y.; Hwang, J.W.; Natarajan, S.B.; Kim, W.S.; Moon, S.H.; Jeon, B.T.; Park, P.J. Antioxidant and anticancer effects of extracts from fermented Haliotis discus hannai with Cordyceps militaris mycelia. Food Sci. Biotechnol. 2016, 25, 1775-1782. [CrossRef]

22. Song, J.; Wang, Y.; Teng, M.; Zhang, S.; Yin, M.; Lu, J.; Liu, Y.; Lee, R.J.; Wang, D.; Teng, L. Cordyceps militaris induces tumor cell death via the caspase-dependent mitochondrial pathway in HepG2 and MCF-7 cells. Mol. Med. Rep. 2016, 13, 5132-5140. [CrossRef]

23. Chen, C.; Wang, M.L.; Jin, C.; Chen, H.J.; Li, S.H.; Li, S.Y.; Dou, X.F.; Jia, J.Q.; Gui, Z.Z. Cordyceps militaris polysaccharide triggers apoptosis and G0/G1 cell arrest in cancer cells. J. Asia Pac. Entomol. 2015, 18, 433-438. [CrossRef]

24. Uhrinová, A.; Pol'ančíková, N. Antioxidant Activity of the Fungus Cordyceps sinensis Grown on Two Different Media. Folia Vet. 2018, 62, 68-73.

25. Wu, H.C.; Chen, S.T.; Chang, J.C.; Hsu, T.Y.; Cheng, C.C.; Chang, H.S.; Liu, C.S.; Wu, Y.C.; Liang, Z.C. Radical Scavenging and Antiproliferative Effects of Cordycepin-Rich Ethanol Extract from Brown Rice- Cultivated Cordyceps militaris (Ascomycetes) Mycelium on Breast Cancer Cell Lines. Int. J. Med. Mushrooms 2019, 21, 657-669. [CrossRef]

26. Hsiao-Ping, K.; Lin, Y.S.; Huang, S.T.; An-Chi, W.; Lai, J.T. Method for Cultivating Cordyceps Militaris Fruiting Body. USA Patent 20190053438A1, 21 February 2019. 
27. Liu, S.C.; Chiu, C.P.; Tsai, C.H.; Hung, C.Y.; Li, T.M.; Wu, Y.C.; Tang, C.H. Soya-cerebroside, an extract of Cordyceps militaris, suppresses monocyte migration and prevents cartilage degradation in inflammatory animal models. Sci. Rep. 2017, 7, 43205. [CrossRef]

28. Chiang, S.S.; Liang, Z.C.; Wang, Y.C.; Liang, C.H. Effect of light-emitting diodes on the production of cordycepin, mannitol and adenosine in solid-state fermented rice by Cordyceps militaris. J. Food Compos. Anal. 2017, 60, 51-56. [CrossRef]

29. Wang, X.; Liu, F.; Li, F.; Cai, H.; Sun, W.; Chen, X.; Gao, H.; Shen, W. Determination of cordycepin content of Cordyceps militaris recombinant rice by high performance liquid chromatography. Trop. J. Pharm. Res. 2016, 15, 2235-2239. [CrossRef]

30. Eguchi, F.; Kalaw, S.P.; Dulay, R.M.R.; Miyasawa, N.; Yoshimoto, H.; Seyama, T.; Reyes, R.G. Nutrient composition and functional activity of different stages in the fruiting body development of Philippine paddy straw mushroom, Volvariella volvacea (Bull.: Fr.) Sing. Adv. Environ. Biol. 2015, 9, 54-66.

31. Olatunji, O.J.; Tang, J.; Tola, A.; Auberon, F.; Oluwaniyi, O.; Ouyang, Z. The genus Cordyceps: An extensive review of its traditional uses, phytochemistry and pharmacology. Fitoterapia 2018, 129, 293-316. [CrossRef]

32. Amirullah, N.A.; Abidin, N.Z.; Abdullah, N. The potential applications of mushrooms against some facets of atherosclerosis: A review. Food Res. Int. 2018, 105, 517-536. [CrossRef]

33. Khan, M.; Tania, M. Cordycepin in Anticancer Research: Molecular Mechanism of Therapeutic Effects. Curr. Med. Chem. 2019. [CrossRef]

34. Tuli, H.S.; Kashyap, D.; Sharma, A.K. Cordycepin: A cordyceps metabolite with promising therapeutic potential. In Fungal Metabolites; Springer: Cham, Switzerland, 2017; pp. 761-782.

35. Kang, N.; Lee, H.H.; Park, I.; Seo, Y.S. Development of High Cordycepin-Producing Cordyceps militaris Strains. Mycobiology 2017, 45, 31-38. [CrossRef]

36. Tian, X.; Li, Y.; Shen, Y.; Li, Q.; Wang, Q.; Feng, L. Apoptosis and inhibition of proliferation of cancer cells induced by cordycepin. Oncol. Lett. 2015, 10, 595-599. [CrossRef]

37. Zheng, J.; Wu, M.; Wang, H.; Li, S.; Wang, X.; Li, Y.; Wang, D.; Li, S. Network Pharmacology to Unveil the Biological Basis of Health-Strengthening Herbal Medicine in Cancer Treatment. Cancers 2018, 10, 461. [CrossRef]

38. Poornima, P.; Kumar, J.D.; Zhao, Q.; Blunder, M.; Efferth, T. Network pharmacology of cancer: From understanding of complex interactomes to the design of multi-target specific therapeutics from nature. Pharmacol. Res. 2016, 111, 290-302. [CrossRef]

39. Fang, J.; Cai, C.; Wang, Q.; Lin, P.; Zhao, Z.; Cheng, F. Systems Pharmacology-Based Discovery of Natural Products for Precision Oncology Through Targeting Cancer Mutated Genes. CPT Pharm. Syst. Pharmacol. 2017, 6, 177-187. [CrossRef]

40. Hu, J.X.; Thomas, C.E.; Brunak, S. Network biology concepts in complex disease comorbidities. Nat. Rev. Genet. 2016, 17, 615. [CrossRef]

41. Camacho, D.M.; Collins, K.M.; Powers, R.K.; Costello, J.C.; Collins, J.J. Next-generation machine learning for biological networks. Cell 2018, 173, 1581-1592. [CrossRef]

42. Park, M.; Park, S.Y.; Lee, H.J.; Kim, C.E. A Systems-Level Analysis of Mechanisms of Platycodon grandiflorum Based on A Network Pharmacological Approach. Molecules 2018, 23, 2841. [CrossRef]

43. Lee, W.Y.; Lee, C.Y.; Kim, Y.S.; Kim, C.E. The Methodological Trends of Traditional Herbal Medicine Employing Network Pharmacology. Biomolecules 2019, 9, 362. [CrossRef]

44. Zhang, R.Z.; Yu, S.J.; Bai, H.; Ning, K. TCM-Mesh: The database and analytical system for network pharmacology analysis for TCM preparations. Sci. Rep. 2017, 7, 2821. [CrossRef]

45. Szklarczyk, D.; Santos, A.; von Mering, C.; Jensen, L.J.; Bork, P.; Kuhn, M. STITCH 5: Augmenting protein-chemical interaction networks with tissue and affinity data. Nucleic Acids Res. 2015, 44, D380-D384. [CrossRef]

46. Kuleshov, M.V.; Jones, M.R.; Rouillard, A.D.; Fernandez, N.F.; Duan, Q.; Wang, Z.; Koplev, S.; Jenkins, S.L.; Jagodnik, K.M.; Lachmann, A. Enrichr: A comprehensive gene set enrichment analysis web server 2016 update. Nucleic Acids Res. 2016, 44, W90-W97. [CrossRef]

47. Ziegler, U.; Groscurth, P. Morphological features of cell death. Physiology 2004, 19, 124-128. [CrossRef]

48. Shannon, P.; Markiel, A.; Ozier, O.; Baliga, N.S.; Wang, J.T.; Ramage, D.; Amin, N.; Schwikowski, B.; Ideker, T. Cytoscape: A software environment for integrated models of biomolecular interaction networks. Genome Rese. 2003, 13, 2498-2504. [CrossRef] 
49. Lipponen, P.; Aaltomaa, S.; Kosma, V.M.; Syrjänen, K. Apoptosis in breast cancer as related to histopathological characteristics and prognosis. Eur. J. Cancer 1994, 30, 2068-2073. [CrossRef]

50. Feng, Y.; Spezia, M.; Huang, S.; Yuan, C.; Zeng, Z.; Zhang, L.; Ji, X.; Liu, W.; Huang, B.; Luo, W. Breast cancer development and progression: Risk factors, cancer stem cells, signaling pathways, genomics, and molecular pathogenesis. Genes Dis. 2018, 5, 77-106. [CrossRef]

51. Crown, J.P. The platinum agents: A role in breast cancer treatment? Semin. Oncol. 2001, 28 (Suppl. 3), 28-37. [CrossRef]

52. Cai, H.; Li, J.; Gu, B.; Xiao, Y.; Chen, R.; Liu, X.; Xie, X.; Cao, L. Extracts of Cordyceps sinensis inhibit breast cancer cell metastasis via down-regulation of metastasis-related cytokines expression. J. Ethnopharmacol. 2018, 214, 106-112. [CrossRef]

53. Lee, H.J.; Burger, P.; Vogel, M.; Friese, K.; Brüning, A. The nucleoside antagonist cordycepin causes DNA double strand breaks in breast cancer cells. Investig. New Drugs 2012, 30, 1917-1925. [CrossRef]

54. Staring, K.E. Comparison of Wild and Cultivated Extracts of Cordyceps Sinensis Apoptotic Potential. 2013. Available online: https://pdfs.semanticscholar.org/b35d/82573910f61b555d0d502be0dc741602f4a8.pdf (accessed on 15 August 2019).

55. Cohen, G.; Sun, X.; Snowden, R.; Dinsdale, D.; Skilleter, D. Key morphological features of apoptosis may occur in the absence of internucleosomal DNA fragmentation. Biochem. J. 1992, 286, 331-334. [CrossRef]

56. Koc, Y.; Urbano, A.; Sweeney, E.; McCaffrey, R. Induction of apoptosis by cordycepin in ADA-inhibited TdT-positive leukemia cells. Leukemia 1996, 10, 1019-1024.

57. Chaicharoenaudomrung, N.; Jaroonwitchawan, T.; Noisa, P. Cordycepin induces apoptotic cell death of human brain cancer through the modulation of autophagy. Toxicol. Vitr. 2018, 46, 113-121. [CrossRef]

58. Zhang, Y.; Zhang, X.X.; Yuan, R.Y.; Ren, T.; Shao, Z.Y.; Wang, H.F.; Cai, W.L.; Chen, L.T.; Wang, X.A.; Wang, P. cordycepin induces apoptosis in human pancreatic cancer cells via the mitochondrial-mediated intrinsic pathway and suppresses tumor growth in vivo. OncoTargets Ther. 2018, 11, 4479. [CrossRef]

59. Zhang, C.; Zhong, Q.; Zhang, X.; Hu, D.; He, X.; Li, Q.; Feng, T. Effects of Cordycepin on Proliferation, Apoptosis and NF-кB Signaling Pathway in A549 Cells. J. Chin. Med. Mater. 2015, 38, 786-789.

60. Chen, Y.H.; Wang, J.Y.; Pan, B.S.; Mu, Y.F.; Lai, M.S.; So, E.C.; Wong, T.S.; Huang, B.M. Cordycepin enhances cisplatin apoptotic effect through caspase/MAPK pathways in human head and neck tumor cells. OncoTargets Ther. 2013, 6, 983.

61. Garber, K. New apoptosis drugs face critical test. Nat. Biotechnol. 2005, 23, 409-411. [CrossRef]

62. Jänicke, R.U. MCF-7 breast carcinoma cells do not express caspase-3. Breast Cancer Res. Treat. 2009, 117, 219-221. [CrossRef]

63. Wang, S.; He, M.; Li, L.; Liang, Z.; Zou, Z.; Tao, A. Cell-in-cell death is not restricted by caspase-3 deficiency in MCF-7 cells. J. Breast Cancer 2016, 19, 231-241. [CrossRef] 